Check for updates

Cite this: Chem. Sci., 2018, 9, 6670

๑ All publication charges for this article have been paid for by the Royal Society of Chemistry

Received 22nd April 2018

Accepted 11th July 2018

DOI: $10.1039 / c 8 s c 01829 d$

rsc.li/chemical-science

\title{
Sensitized triplet-triplet annihilation upconversion in water and its application to photochemical transformations $\uparrow$
}

\author{
Christoph Kerzig (DD * and Oliver S. Wenger (D) *
}

Sensitized triplet-triplet annihilation (TTA) is a promising mechanism for solar energy conversion, but so far its application has been practically completely limited to organic solvents and self-assembled or solid state systems. Combining water-soluble ruthenium complex-pyrene dyads with particularly long excited-state lifetimes as sensitizers and highly fluorescent commercial anthracenes as acceptors/ annihilators, we were able to achieve green-to-violet upconversion with unprecedented quantum yields in pure water. Compared to the only known system exploiting sensitized TTA in homogeneous aqueous solution, we improve the overall photon upconversion efficiency by a full order of magnitude and present the very first example for a chemical transformation on a laboratory scale via upconversion in water. Specifically, we found that a thermodynamically challenging carbon-chlorine bond activation can be driven by green photons from an inexpensive continuous wave light source in the presence of dissolved oxygen. Our study is thus potentially relevant in the context of cleaning water from halogenated (toxic) contaminants and for sustainable photochemistry in the most environmentally friendly solvent.

\section{Introduction}

Sensitized triplet-triplet annihilation (TTA) is an important phenomenon because it permits not only a viable access to photochemical upconversion - the process of transforming lowenergy photons into radiation of higher energy ${ }^{1-7}-$ but also the indirect formation of high-energy singlet states for chemical reactions driven by visible light. ${ }^{, 8-11}$ Compared to the related approach of absorbing two photons simultaneously, ${ }^{12}$ sensitized TTA-based technologies do not necessarily require extremely high light intensities as provided by pulsed lasers, ${ }^{13}$ so they are much more promising for solar energy harvesting.

Upconversion through sensitized TTA was discovered about half a century ago $^{\mathbf{1 4}}$ and numerous sensitizer-acceptor/ annihilator couples have been identified both in organic solvents and in solid matrices. ${ }^{1-7}$ However, it was not until very recently that the first proof of principle for upconversion in homogeneous aqueous solution was provided, ${ }^{15,16}$ but the quantum efficiency was yet very low. Two main challenges make upconversion in water tricky to achieve. First, acceptor/ annihilator molecules usually have a strongly pronounced non-polar character inherently making them insoluble in water.

Department of Chemistry, University of Basel, St. Johanns-Ring 19, 4056 Basel, Switzerland.E-mail: christoph.kerzig@unibas.ch; oliver.wenger@unibas.ch $\dagger$ Electronic supplementary information (ESI) available: Comprehensive experimental details, synthetic procedures and additional details on all experiments. See DOI: 10.1039/c8sc01829d
Second, a highly polar solvent favours electron transfer reactions, which can compete with the desired triplet-triplet energy transfer (TTET) between the sensitizer and the acceptor/ annihilator.

Since the discovery of the sensitized anthracene photodimerization in $2006,{ }^{8}$ there has been an ongoing interest in driving photochemical reactions via sensitized TTA, because this process allows the use of visible photons to initiate reactions, which would otherwise require UV light. Examples in organic solvents, ${ }^{3,8,9}$ gels ${ }^{10}$ and supramolecular structures ${ }^{11}$ have already been reported, but there is no example in homogeneous aqueous solution. In the context of sustainable chemistry, chemical reactions through sensitized TTA in homogeneous aqueous solution, i.e., in the cheapest and "greenest" solvent, ${ }^{17,18}$ are highly desirable.

The structures of the key compounds employed in this study and the corresponding energy diagram of the sensitized TTA mechanism are shown in Scheme 1. Beyond mechanistic investigations leading to a drastic improvement of upconversion quantum efficiencies in water, the high reactivity of the singlet states so accessible is exploited for a laboratory-scale carbon-chlorine bond activation that operates under very attractive conditions in aerated solution. For conciseness, experimental details and synthetic procedures have been relegated to the ESI. $\uparrow$ As we will show by addressing both photon upconversion and chemical transformations, sensitized TTA in pure water is a promising approach for the photochemistry of the 21 st century. 

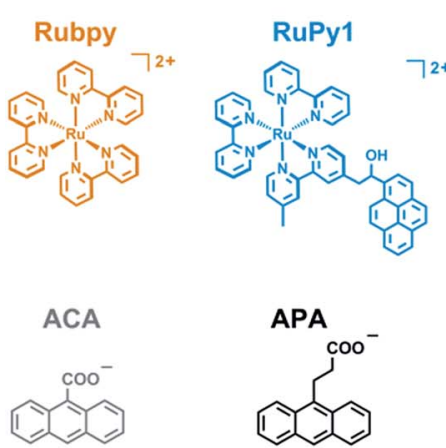

APA

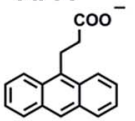

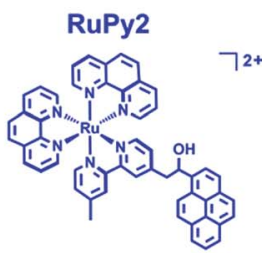

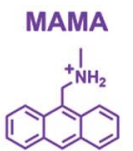

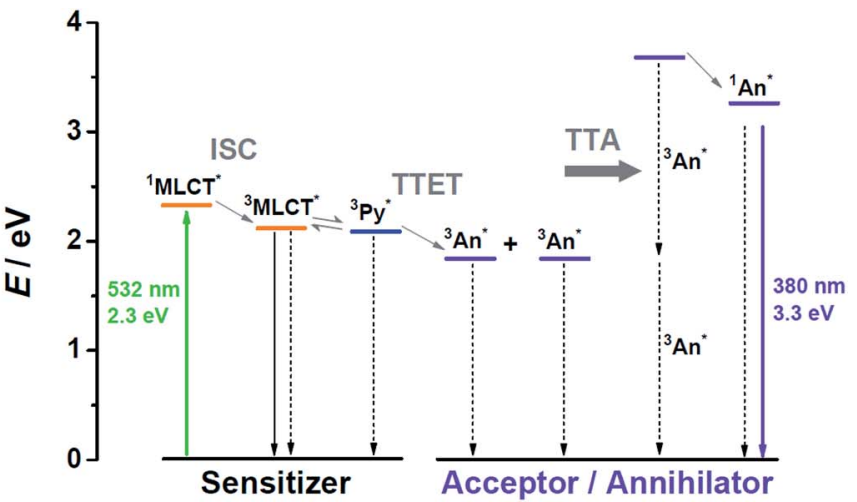

Scheme 1 (Left) Molecular structures and abbreviations of the water-soluble sensitizers (upper row) and acceptors/annihilators (lower row) investigated in this work. (Right) Qualitative energy diagram illustrating upconversion through sensitized TTA using a Ru-based sensitizer (whose MLCT-excited state is in equilibrium with the pyrene (Py) triplet in the dyads ${ }^{19}$ ) and an anthracene derivative (An) as the acceptor/annihilator. Excited state energies were taken from ref. 20. For further explanations, see the main text.

\section{Results and discussion}

\subsection{Sensitizers}

In upconversion compositions, the sensitizers are typically employed at very low concentrations (micromolar), so they can be tailored for optimum properties with little regard to their cost. Inspired by the frequent observation of the so-called triplet reservoir effect ${ }^{19,21}$ of ruthenium complex-pyrene bichromophores in organic solvents, which significantly prolongs the excited-state lifetimes while maintaining triplet formation quantum yields very close to unity, ${ }^{22,23}$ we designed two dyads RuPy1 and RuPy2 (Scheme 1) such that they are sufficiently water-soluble, even as readily accessible hexafluorophosphate salts. For this purpose, we used only one pyrene-containing diimine ligand, ${ }^{22}$ which also bears an additional polar group with favourable hydrogen-bonding properties. The synthetic procedures for the preparation of the dyads RuPy1 and RuPy2 are given in Section 2 of the ESI. $\dagger$

Fig. 1 compares the UV-Vis absorption (solid lines) and luminescence spectra (dotted lines) of the dyads with those of the reference compounds Rubpy as well as pyrene-1-

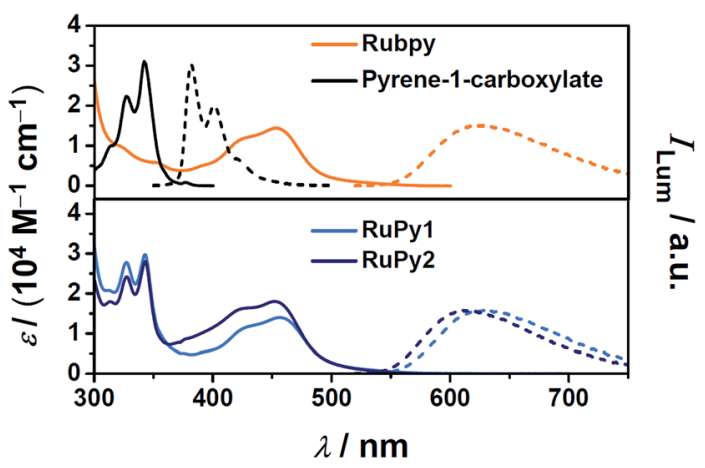

Fig. 1 Calibrated UV-Vis absorption (solid lines) and normalized luminescence (dotted lines) spectra (upon excitation at $500 \mathrm{~nm}$ and $330 \mathrm{~nm}$ for the complexes and the pyrene reference, respectively) of reference compounds (upper panel) and metal complex-arene dyads (lower panel) in homogeneous aqueous solution. carboxylate, i.e., with the isolated water-soluble chromophores. Inspection of the absorption spectra clearly shows that the dyads possess both the typical MLCT absorption ${ }^{24}$ that is centered at about $450 \mathrm{~nm}$ tailing to the yellow-green region of the spectrum and the fine-structured near-UV absorption of the

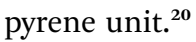

After selective excitation into the MLCT absorption band of the dyads, characteristic ${ }^{3}$ MLCT phosphorescence ${ }^{24}$ is observed (Fig. 1). Compared to Rubpy and RuPy1, the position of the emission band of RuPy2 is blue-shifted by about $15 \mathrm{~nm}$, which is due to the higher energy of the ${ }^{3}$ MLCT state when phenanthroline ligands are present. ${ }^{25,26}$ The luminescence quantum yields of Rubpy and RuPy1 are quite similar; RuPy2, however, is more strongly emissive by about $50 \%$. All pertinent properties extracted from the steady-state measurements are summarized, together with other sensitizer-related properties, in Table 1.

Fig. 2 juxtaposes the transient absorption and emission properties of the three sensitizers upon excitation with green (532 nm) laser pulses. The transient absorption (TA) spectrum of RuPy1 still has characteristic signatures of the Rubpy triplet with a bleach at $455 \mathrm{~nm}$ and a prominent absorption shoulder around $370 \mathrm{~nm}$ (vertical lines in the left part of Fig. 2), while that of RuPy2 is practically identical to the recently recorded triplettriplet absorption spectrum of pyrene-1-carboxylate in water. ${ }^{27}$ The TA spectra of all sensitizers show negative signals in the red, which are assigned to ${ }^{3}$ MLCT emission. Under conditions of identical detection sensitivity, this emission is weaker for the two dyads than for Rubpy. The time-resolved signals of the three sensitizers (panels d-f in Fig. 2) decay monoexponentially with identical rate constants of absorption and emission traces $( \pm 5 \%)$. A key finding is that excited-state lifetime $\left(\tau_{0}\right)$ extensions by up to a factor of 30 have been achieved for the dyads (compared to $0.6 \mu$ s for Rubpy). Specifically, for RuPy1 we find $\tau_{0}$ $=2.6$ and for RuPy2 $\tau_{0}=17.9 \mu$ s (Table 1 ). The lifetime measurements were carried out at low laser intensities to avoid self-quenching that might occur when high concentrations of long-lived triplet states are produced. As a result of the variation of the ${ }^{3}$ MLCT energy when different diimine co-ligands are 
Table 1 Selected photochemical/-physical properties of the sensitizers used in this study

\begin{tabular}{|c|c|c|c|c|c|c|c|}
\hline Sensitizer & $\varepsilon_{532} /\left(\mathrm{M}^{-1} \mathrm{~cm}^{-1}\right)^{a}$ & $\lambda_{\max }(\mathrm{em}) / \mathrm{nm}^{b}$ & $\tau_{0} / \mu \mathrm{s}^{c}$ & $\phi_{\mathrm{em}}{ }^{d}$ & $\alpha^{e}$ & $k_{\text {oxygen }} /\left(\mathrm{M}^{-1} \mathrm{~s}^{-1}\right)^{f}$ & $k_{\text {TTET }} /\left(\mathrm{M}^{-1} \mathrm{~s}^{-1}\right)^{g}$ \\
\hline Rubpy & 720 & 625 & 0.6 & 0.063 (ref. 31) & - & $3.2 \times 10^{9}$ & $1.4 \times 10^{9}$ \\
\hline RuPy1 & 990 & 630 & 2.6 & 0.058 & 0.79 & $2.3 \times 10^{9}$ & $2.3 \times 10^{9}$ \\
\hline
\end{tabular}

${ }^{a}$ Molar absorption coefficients of the sensitizers at the excitation wavelength for upconversion (532 nm). ${ }^{b}$ Maxima of MLCT emission in $\mathrm{H}_{2} \mathrm{O}$. ${ }^{c}$ Unquenched lifetimes of excited sensitizers as determined by the average of absorption and luminescence decay kinetics. ${ }^{d}$ Luminescence quantum yields of the MLCT emission using the value of Rubpy as the reference. ${ }^{e}$ Fraction of pyrene-like triplets in the excited-state equilibrium. ${ }^{f}$ Rate constants of oxygen quenching (with $0.27 \mathrm{mM}$ of dissolved oxygen in air-saturated water). ${ }^{20}{ }^{g}$ Rate constants of triplet-triplet energy transfer (TTET) reactions between excited sensitizers and the anthracene derivative MAMA.
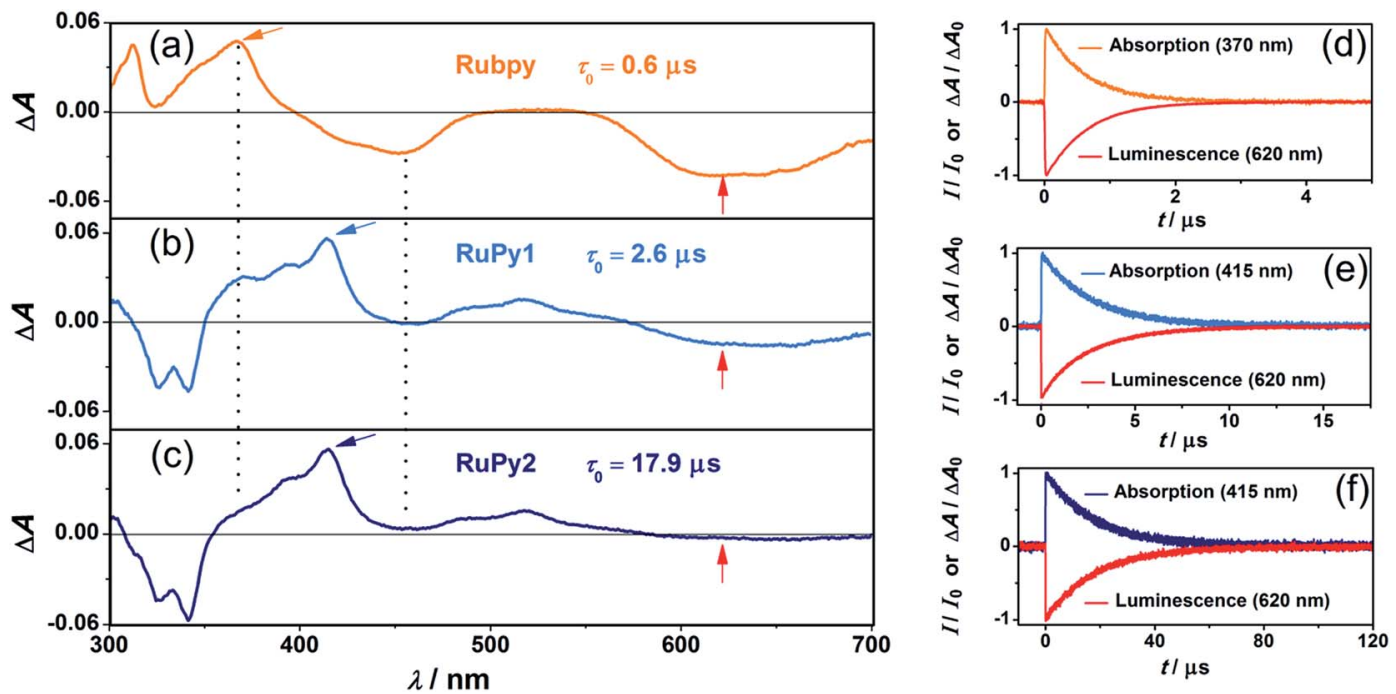

Fig. 2 Excited-state properties of the sensitizers in Ar-saturated aqueous solution (Rubpy, $30 \mu \mathrm{M}$, panels a and d; RuPy1, $22 \mu \mathrm{M}$, panels b and e; RuPy2, $28 \mu \mathrm{M}$, panels $\mathrm{c}$ and f) upon excitation with green $(532 \mathrm{~nm}$ ) laser pulses of $c a$. $10 \mathrm{~ns}$ duration. Panels a-c contain transient absorption spectra (immediately following excitation with $45 \mathrm{~mJ}$ pulses and time-integrated over $200 \mathrm{~ns}$ ). Arrows indicate the detection wavelengths for the kinetic measurements (with laser pulse energies of $16 \mathrm{~mJ}$ ) shown in panels $d-f$. For further explanations, see text.

used, the excited-state lifetimes of RuPy1 and RuPy2 differ by almost one order of magnitude although the same pyrenecontaining ligand is employed. All these results obtained for the new water-soluble bichromophores are in line with a fast and reversible excited-state equilibrium between ${ }^{3}$ MLCT and ${ }^{3}$ pyrene..$^{22,28-30} \mathrm{~A}$ simple quantity characterizing this equilibrium is provided by the so-called $\alpha$ value, ${ }^{28}$ which is the fraction of pyrene-like triplets in the equilibrium distribution. With the unquenched lifetimes of isolated ${ }^{3}$ Rubpy and ${ }^{3}$ pyrene-1carboxylate, ${ }^{27}$ we were able to estimate this value ${ }^{28}$ from the measured excited-state lifetimes of the two dyads (Table 1).

The emissive excited states of all sensitizers are quenched by dissolved oxygen with rate constants close to the diffusion limit (see also Section 6 of the ESI $\dagger$ ). The dyad RuPy2 compares even very favourably (with respect to the emission quantum yield and oxygen quenching efficiency) with all known Ru-based oxygen sensors used in aqueous media. ${ }^{32-38}$ Moreover, the non-polar character of the pyrene-containing ligand should facilitate the cellular uptake, ${ }^{36}$ thus making RuPy2 a promising candidate for bioimaging.

The change of the excited-state character from ${ }^{3}$ MLCT (Rubpy) to ${ }^{3}$ pyrene (RuPy1 and RuPy2) increases the rate constant of the desired energy transfer between the respective sensitizer and MAMA by roughly 50\% (see the last column of Table 1 for the rate constants and Section 3.1 of the ESI for details $\dagger$ ). Since MAMA is a cationic anthracene-based acceptor/ annihilator, the increase in $k_{\text {TTET }}$ is most likely due to a reduced coulombic repulsion when the uncharged pyrene unit rather than the cationic $\mathrm{Ru}(\mathrm{II})$ moiety passes the triplet energy.

The long excited-state lifetimes of the metal complex-arene dyads, combined with their higher (kinetic) reactivity for TTET, are highly beneficial for photon upconversion systems, as we will show in Section 2.3.

\subsection{Acceptors/annihilators}

We focused on anthracene-based acceptors/annihilators as they have ideal thermodynamic properties for the desired sensitized TTA mechanism and - as opposed to pyrene derivatives - they usually do not suffer from the intrinsic problem of energywasting direct excimer formation. However, most of the watersoluble anthracene derivatives that have already been characterized photochemically possess rather low fluorescence quantum yields ${ }^{15,20,39-41}$ (associated with short excited-state lifetimes) and high intersystem crossing efficiencies. A 
possible explanation for this unfavourable behavior is provided by their chemical structures; the heteroatoms are in very close proximity to the anthracene chromophore, which should increase the intersystem crossing rates. We speculated that the separation of the ionic group (facilitating the solubility in water) by short alkyl spacers from the anthracene core might result in improved properties for photon upconversion. To test this hypothesis, we selected the ionic forms of two commercially available anthracenes (APA and MAMA; see Scheme 1 for the structures), and first compared their steady-state properties as well as fluorescence lifetimes with those of the previously used $\mathbf{A C A}^{\mathbf{1 5}}$ as the reference (Fig. 3).

The absorption and fluorescence spectra of all water-soluble anthracene derivatives under study are quite similar. However, the separation of the heteroatom(s)/ionic groups from the anthracene skeleton by short alkyl spacers significantly increases both the fluorescence lifetime and quantum yield (insets in Fig. 3).

Fig. 4 shows the indirect generation of the anthracene derivative triplet states through TTET using the sensitizers characterized in Section 2.1. After the decay of sensitizer triplets, all transient absorption spectra only consist of a fine-structured absorption band peaking at about $425 \mathrm{~nm}$ (main plot of Fig. 4), which is in perfect agreement with triplet anthracene absorption spectra previously recorded in polar solvents. ${ }^{42}$ Furthermore, the spectra clearly show that photoinduced electron transfer reactions do not compete with the desired TTET, because the former would manifest themselves by intense absorption signals of the anthracene radical ions in the red. ${ }^{\mathbf{4 3 , 4 4}}$ Triplet anthracene spectra of ACA and MAMA are independent of the sensitizer used, but in the case of APA, we could only employ Rubpy as the sensitizer, because the bichromophoric triplet energy donors are almost completely insoluble in the presence of APA, which most likely serves as a crystallization-inducing counterion. These observations illustrate some of the difficulties in identifying watersoluble sensitizer-acceptor/annihilator couples.

Since the precise knowledge of the triplet anthracene concentration during our measurements is required for

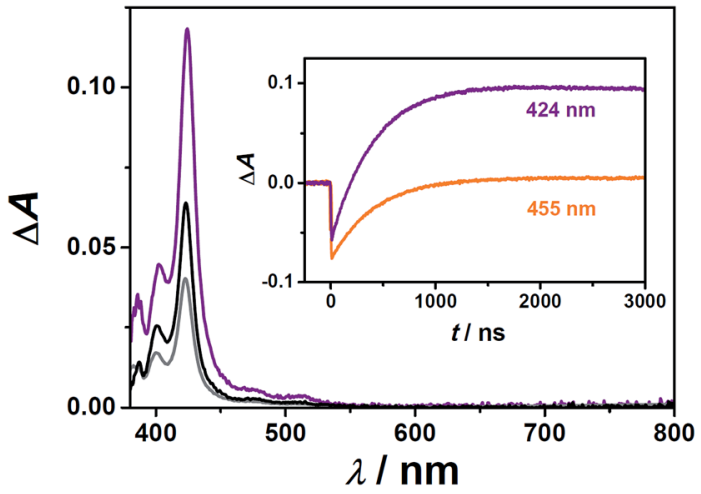

Fig. 4 Triplet-triplet energy transfer from ${ }^{3}$ Rubpy (orange) to the anthracene derivatives MAMA (violet), APA (black) and ACA (gray) in deoxygenated aqueous solutions upon excitation of the sensitizer $(30 \mu \mathrm{M})$ with $532 \mathrm{~nm}$ laser pulses $(37 \mathrm{~mJ})$. Main plot, transient absorption spectra after sensitizer excited-state decay with MAMA (0.82 mM), APA (0.28 mM), or ACA (1 mM) (recorded by time integration over a detection period of $200 \mathrm{~ns}$ with a time delay of $3 \mu \mathrm{s})$. For clarity, the spectra with APA and ACA have been multiplied by $1 / 2$ and $1 / 5$, respectively. Inset, comparative kinetic traces for the system Rubpy/MAMA (0.54 mM) at the maxima of Rubpy bleaching (455 nm) and ${ }^{3}$ MAMA absorption (424 nm). For further details, see text.

characterizing the TTA kinetics, we calibrated the triplet-triplet absorption spectra of APA and MAMA employing relative actinometry with the widely used reference compound Rubpy. ${ }^{20,45,46}$ To this end, we first analyzed the initial Rubpy ground-state bleaching $\left(\Delta \varepsilon_{455}=-10100 \mathrm{M}^{-1} \mathrm{~cm}^{-1}\right)^{46}$ and the triplet anthracene formation kinetics from laser flash photolysis (LFP) experiments on the very same solution (example traces are displayed in the inset of Fig. 4); based on the known energy transfer efficiency at a given concentration of the respective anthracene derivative, we calculated the molar absorption coefficient at the maximum of the triplet anthracene spectrum, $424 \mathrm{~nm}$. Moreover, the fraction of anthracene triplets that already decayed on the timescale of the TTET reaction was considered $^{47}$ to avoid a small but systematic error. Three independent determinations at different concentrations were
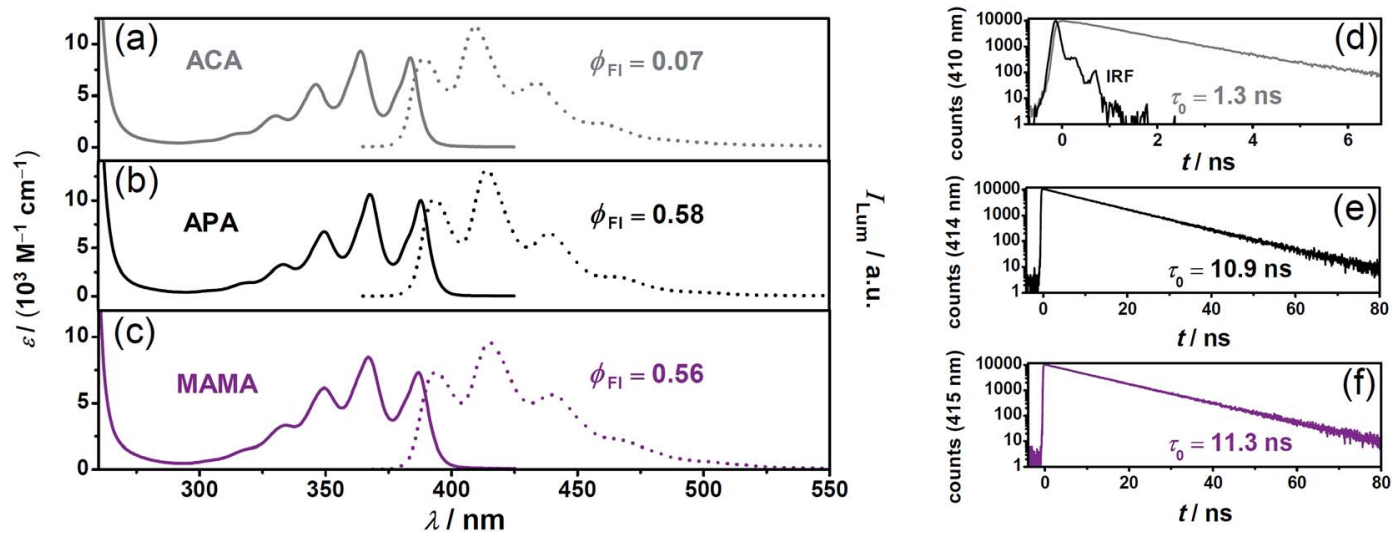

Fig. 3 Ground-state and singlet-excited state properties of the acceptors/annihilators, which were investigated in this study (the same colour code for the compounds as in Scheme 1). Panels a-c, calibrated UV-Vis absorption (solid lines) and normalized luminescence (dotted lines) spectra. Panels $d-f$, unquenched excited-singlet state lifetimes measured at the respective emission maxima $\left(\lambda_{\text {ex }}=375 \mathrm{~nm}\right)$. The instrument response function (IRF) is included in panel d. For details, see text and page S2 of the ESI. $\dagger$ 
carried out for both anthracene derivatives and the results have been compiled in Table 2 .

Finally, the triplet decay analyses of the anthracene derivatives (Section 3.2 of the ESI $\dagger$ ) revealed long unquenched lifetimes and TTA rate constants close to the diffusion limit (Table 2). These properties, and the absence of electron transfer quenching with the excited sensitizers, make APA and MAMA very promising candidates for upconversion-based applications in homogeneous aqueous solution.

\subsection{Photon upconversion in water}

Quasi-quantitative quenching of the excited sensitizer through TTET is essential for efficient photon upconversion, but typically requires millimolar concentrations of the energy acceptor, which might cause solubility problems, especially in water. Longer sensitizer triplet lifetimes are therefore highly beneficial, because they permit the use of lower acceptor concentrations. The improvement achieved when metal complex-arene dyads are employed instead of conventional Rubpy is demonstrated in Fig. 5(a). Under standardized conditions using $0.25 \mathrm{mM}$ of the acceptor MAMA and producing identical excited-state concentrations of the respective sensitizer, the energy transfer efficiency monitored by the ${ }^{3}$ MAMA absorption at $424 \mathrm{~nm}$ is significantly higher for the upconversion systems containing the dyads (blue traces in Fig. 5(a)), and amounts to more than $90 \%$ when the dyad with the longest excited-state lifetime, RuPy2, is used (calculated with the parameters presented in Table 1 and the well-established photokinetic equations). Owing to the quadratic dependence of the upconversion emission on the initial triplet concentration of the acceptor/annihilator, the triplet lifetime of the sensitizer has a particularly important influence on the upconversion efficiency. The inset of Fig. 5(a) illustrates this effect enabling an improvement by more than one order of magnitude. This highly successful approach with long-lived bichromophores as sensitizers $^{\mathbf{2 , 4 , 4 8 - 5 2}}$ was hitherto unreported in water.

Besides the economic factor, the concept of high TTET efficiencies at low acceptor concentrations has two further advantages. First, parasitic reabsorption of the upconverted light by the ground-state acceptor/annihilator does not occur at the short-wavelength edge of the fluorescence spectrum allowing unhindered emission of the most highly upconverted light (an example of this effect is given in Fig. S4 $\dagger$ ). Second, destructive self-quenching of the singlet-excited acceptor/annihilator ${ }^{8}$ plays only a minor role at low concentrations, which thus creates the basis for high system stabilities.

The effect of the acceptor/annihilator on the delayed fluorescence using standardized conditions is shown in Fig. 5(b). In this series of experiments, Rubpy was the sensitizer and the intensities of the green laser pulses were adjusted such that

Table 2 Upconversion-related properties of the anthracene-derived acceptors/annihilators investigated in this study

\begin{tabular}{lllllll}
\hline Acceptor/Annihilator & $E_{0-0} / \mathrm{eV}^{a}$ & $\phi_{\mathrm{Fl}}{ }^{b}$ & $\tau_{0}\left(\mathrm{~S}_{1}\right) / \mathrm{ns}^{c}$ & $\tau_{0}\left(T_{1}\right) / \mu \mathrm{s}^{d}$ & $\varepsilon_{\max }\left(T_{1}\right) /\left(\mathrm{M}^{-1} \mathrm{~cm}^{-1}\right)^{e}$ & $k_{\mathrm{TTA}} /\left(10^{9} \mathrm{M}^{-1} \mathrm{~s}^{-1}\right)^{f}$ \\
\hline ACA & 3.21 & $0.07^{15,39}$ & 1.3 & $52.9^{15}$ & $39000(420)^{15}$ & 6.8 \\
APA & 3.18 & 0.58 & 10.9 & $62.7 \pm 3.7$ & $47300 \pm 200(424)$ & $2.5 \pm 0.4$ \\
MAMA & 3.18 & 0.56 & 11.3 & $99.6 \pm 2.8$ & $39400 \pm 1800(424)$ & $2.7 \pm 0.3$
\end{tabular}

${ }^{a}$ Energy of the excited singlet states determined from the intersections of absorption and fluorescence spectra. ${ }^{b}$ Fluorescence quantum yields measured with that of ACA as the standard. ${ }^{c}$ Unquenched lifetimes of the excited singlet and ${ }^{d}$ triplet states. ${ }^{e}$ Molar absorption coefficients at the maxima (wavelengths presented in brackets) of triplet-triplet absorption spectra. ${ }^{f}$ Rate constants of triplet-triplet annihilation.
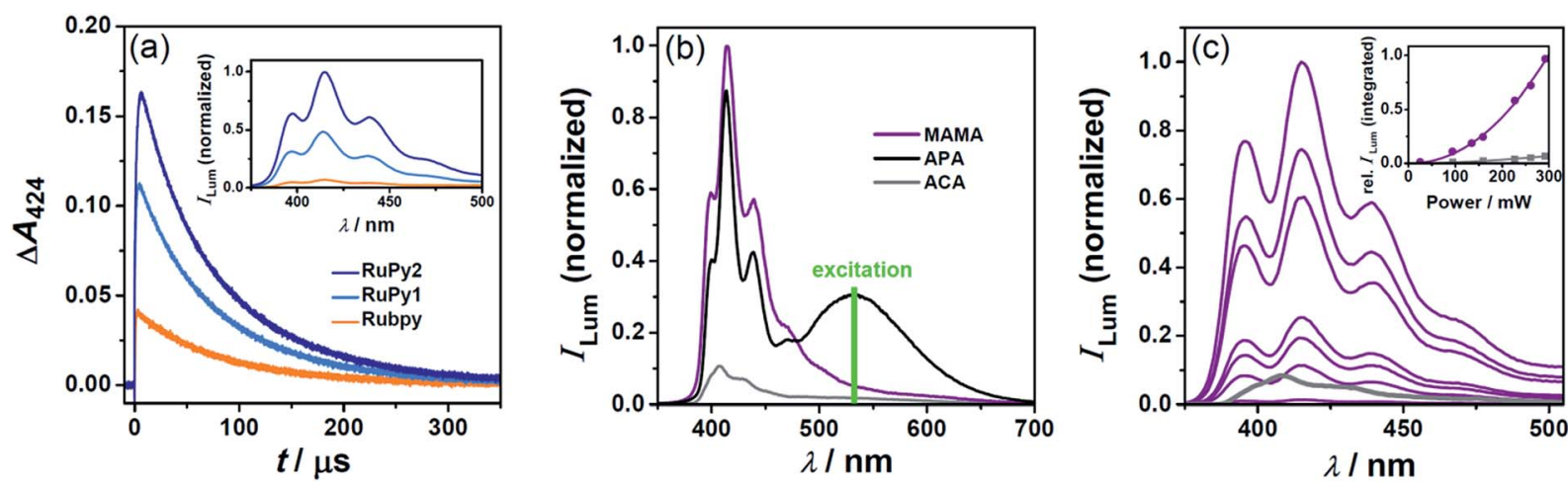

Fig. 5 Photon upconversion in deoxygenated water using a pulsed laser (panels a and b) or a cw light source (panel c) for excitation at $532 \mathrm{~nm}$. (a) Main plot, MAMA ( $0.25 \mathrm{mM})$ triplet formation and decay with the three different sensitizers. For all experiments, initial absorbance $(0.022$ per $\mathrm{cm})$ of the respective sensitizer at $532 \mathrm{~nm}$ and laser intensity $(33 \mathrm{~mJ}$ ) were identical. Inset, time integrated (over $400 \mu \mathrm{s}$ starting with the laser pulse) upconversion emission under the conditions of the main plot. (b) Delayed luminescence spectra employing $30 \mu \mathrm{M}$ Rubpy and $1 \mathrm{mM}$ of the respective acceptor/annihilator. The laser energies were adjusted such that the initial concentrations of anthracene triplets were identical in all cases. The removal of remaining Rubpy phosphorescence from the spectrum was carried out as in Fig. S5 (ESI + ). (c) Power dependences of the

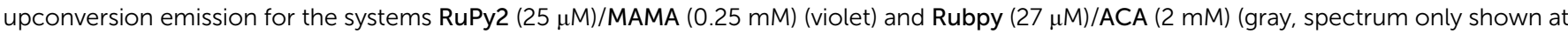
the highest laser power used). 
identical initial concentrations of the three anthracene triplets are produced. The delayed fluorescence spectra were timeintegrated over a period long enough for detecting the complete emission. Compared to the reference system Rubpy/ ACA, which was the most efficient upconversion composition presented in ref. 15, the photon upconversion efficiency is enhanced by about one order of magnitude when using MAMA. With APA as a replacement, however, we observed broad-band visible light generation due to additional excimer fluorescence (black traces in Fig. 5(b)). The ratio of the monomer-to-excimer fluorescence is independent of the APA concentration (see Fig. S4 of the ESI $\dagger$ ). Our control experiments (Section 4 of the ESI $\dagger$ ) suggest that this is a result of the special geometry of the TTA encounter complex. This finding is quite unusual for anthracenes, ${ }^{53,54}$ but potentially important in the context of white-light generation. ${ }^{55,56}$

To demonstrate that our improved upconversion composition does not require high-energy pulsed laser excitation, we employed an inexpensive continuous wave (cw) light source. For comparability with LFP experiments, the same excitation wavelength (532 nm) was chosen and very low sensitizer concentrations $(<30 \mu \mathrm{M})$ were used. The upconversion power dependences measured for our most successful system, RuPy2/MAMA, and the reference system Rubpy/ACA, both under conditions of identical sensitizer excitation as well as 90\% TTET efficiency, are compared in Fig. 5(c). The photon flux dependences of the integrated delayed luminescence so obtained clearly show a non-linear behavior. Their fits with a power function yielded exponents of 2.1 (RuPy2/MAMA) and 1.9 (Rubpy/ACA), respectively; these results are very close to the theoretical value for upconversion through sensitized TTA confirming the mechanism presented in Scheme 1. ${ }^{57,58}$ By control experiments without sensitizers, we also established that interfering luminescences do not contribute to the upconversion signals in any way. Under the comparative conditions of Fig. 5(c), the overall upconversion efficiency of our new system is higher by one order of magnitude. Moreover, delayed emission in the recently published reference system is almost undetectable when an anthracene concentration as low as in the case here $(0.25 \mathrm{mM})$ is employed. This is due to the low TTET efficiency when short-lived ${ }^{3}$ Rubpy is used as a triplet energy donor.

An upconversion quantum yield estimation for the system RuPy2/MAMA (Section 5 of the ESI $\dagger$ ) was carried out using LFP experiments and gave a quantum yield of about $1 \%$. Additional measurements on the ${ }^{3}$ MAMA formation and decay revealed a TTET efficiency of 0.91 and a TTA efficiency of 0.39 (obtained from the $\beta$ value of the fit function shown in Section 3.2 of the ESI $\dagger$ ) under these well-defined conditions. Assuming a theoretical maximum of $11.1 \%$ (ref. 1) (based on the spin-statistical factor $f=0.111$ and an upconversion quantum yield maximum of $100 \%$ ) and the experimentally determined efficiencies/quantum yields for each reaction step in eqn (1), ${ }^{\mathbf{4 , 6}}$ the (theoretical) upconversion quantum yield is calculated (eqn (1)) to be $2.2 \%$, i.e., more than twice as high as our experimental value.

$$
\phi_{\mathrm{UC}}=f \times \phi_{\mathrm{ISC}} \times \phi_{\mathrm{TTET}} \times \phi_{\mathrm{TTA}} \times \phi_{\mathrm{F}}
$$

A possible explanation for this discrepancy is provided by the disproportionation of two anthracene triplets, which is thermodynamically feasible even in organic solvents ${ }^{20}$ and should be favoured in highly polar water. However, we did not observe any signature of the anthracene radical ions in the transient absorption spectra recorded at several time delays, although both aryl radical anions and cations are quite stable in aqueous solution. ${ }^{\mathbf{5 4 5 9}}$ If such a disproportionation reaction represents a deactivation channel, almost instantaneous radical recombination is expected to take place.

\subsection{Reactivity and applications of the excited anthracene derivatives}

With the insights gained in the preceding sections, the use of sensitized TTA couples for chemical reactions in homogeneous aqueous solution became a realistic target.

Kinetically, the excited-singlet states of MAMA and APA are very promising for substrate activation via bimolecular reactions, because they are longer-lived than excited ACA by almost one order of magnitude (Table 2). Given a potential of $+1.2 \mathrm{~V} v s$. NHE for the oxidation of 9-methylanthracene, ${ }^{20}$ the basic structure of APA and MAMA, and their excited-state energies (Table 2), a potential of about $-2 \mathrm{~V} v$ s. NHE is expected for the redox couple anthracene radical cation/singlet-excited anthracene. Regarding their ability to reduce suitable substrates, the excited anthracenes should thus be as reactive as the carbon dioxide radical anion, for which a reduction potential in the range between -1.9 and $-2.0 \mathrm{~V} v s$. $\mathrm{NHE}^{60}$ has been reported. Recently, the high reactivity of $\mathrm{CO}_{2}{ }^{-}$was exploited for the reductive activation $\left(k=3.2 \times 10^{8} \mathrm{M}^{-1} \mathrm{~s}^{-1}\right)$ of trichloroacetate TCA $^{-} \cdot{ }^{61}$ Being a typical chloro-organic substance, $\mathrm{TCA}^{-}$almost instantaneously dissociates upon one-electron reduction to yield a carbon-centered radical and chloride ions. As we found by fluorescence quenching studies with near-UV excitation of APA directly producing ${ }^{1}$ APA* $^{*}$ (Section 7 of the ESI $\dagger$ ), the rate constant of $\mathrm{TCA}^{-}$activation is 2.5 times faster for (monoanionic) ${ }^{1}$ APA* than for $\mathrm{CO}_{2}{ }^{*-}$. Given the same repulsive Coulomb contribution to the reaction rate for the two strongly reducing species, ${ }^{1} \mathbf{A P A} *$ is in fact more reactive than $\mathrm{CO}_{2}{ }^{-}$, and its reduction potential should be slightly more negative than $-2.0 \mathrm{~V} v s$. NHE.

In order to test the applicability of ${ }^{1} \mathbf{A P A}^{*}$ for the laboratoryscale dehalogenation of $\mathrm{TCA}^{-}$in aqueous solution, we performed irradiation experiments in fluorescence cuvettes $(3 \mathrm{~mL})$ and quantified the chloride ions generated in the course of the reaction using a chloride-sensitive electrode (see Section 7 of the ESI $\dagger$ for detailed protocols). We regard this procedure as more reliable than monitoring the organic reaction products, since this radical reaction is likely to produce several anthracene and $\mathrm{TCA}^{-}$derived products, possibly in different hydrolysis or protonation forms.

Upon direct excitation of APA with high-energy photons $(\lambda<$ $400 \mathrm{~nm}$ ), we detected chloride concentrations corresponding to a $\mathrm{TCA}^{-}$dehalogenation of up to $81 \%$ under optimized conditions (Table S3, $\dagger$ entry 6), whereas control experiments established that $\mathrm{TCA}^{-}$is completely stable in our solutions when no 
light source is used (Table S3, $\dagger$ entries 2 and 9), in line with the expectation for recalcitrant chloro-organics. Therefore, the mono-dechlorination of TCA $^{-}$by singlet-excited APA is a wellsuited model reaction.

As opposed to Section 2.3, in our experiments on chemical transformations through sensitized TTA, a millimolar concentration of the anthracene annihilator is required, because this compound is converted into a stoichiometric reducing agent. Hence, there is no need for tailor-made sensitizers with longlived excited states, and we simply used commercial Rubpy. In the presence of this sensitizer, which also has the function of a catalyst in the chemical transformation, we were able to achieve up to $43 \% \mathrm{TCA}^{-}$conversion (Table S3, $\dagger$ entry 13 ) and a turn-over-number TON of up to 15 (Table S3, $\dagger$ entry 12) using a cw laser for selective excitation of Rubpy. All control experiments (see Section 7 of the ESI $\dagger$ ) unambiguously identify the singlet-excited state of APA produced through sensitized TTA as the reacting species. The proposed mechanism of the $\mathrm{TCA}^{-}$ dechlorination, together with pertinent conditions and the optimized yield, is displayed in Scheme 2. It is worth emphasizing that simultaneous two-photon absorption (TPA ${ }^{12}$ does not play any role under our irradiation conditions (Table S3, $\dagger$ entry 10) demonstrating the intrinsic advantage of sensitized TTA-based upconversion over the TPA mechanism.

Surprisingly, our control experiments revealed that dissolved oxygen has only a minor influence on the $\mathrm{TCA}^{-}$dechlorination. We directly monitored an oxygen-scavenging effect using transient absorption spectroscopy on the Rubpy/APA system (see Section 7 of the ESI $\dagger$ ). A rationalization of this effect is provided by the production of ${ }^{1} \mathrm{O}_{2}$ through energy transfer from the APA triplet, either formed upon direct excitation of the anthracene derivative $^{20}$ or via TTET. The singlet oxygen so obtained is known to produce endoperoxides with ground-state anthracenes, ${ }^{62}$ thereby removing oxygen from the solution. Sacrificially using a fraction of acceptor/annihilator molecules for oxygen removal seems to be inapplicable at first glance. However, the very low oxygen solubility in water $(0.27 \mathrm{mM})$ compared to that in typical organic solvents (about $2 \mathrm{mM})^{20}$ renders this oxygeninduced loss of the acceptor/annihilator unimportant under synthetically relevant conditions.

The $\mathrm{TCA}^{-}$dechlorination with the Rubpy/APA system presented in this section is the very first example for a sensitized

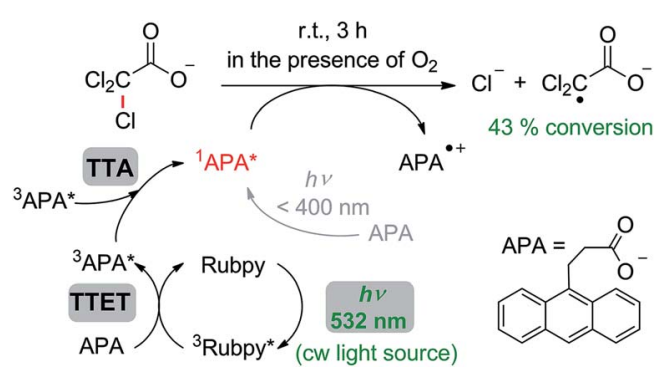

Scheme 2 Reductive monodechlorination of trichloroacetate (TCA ${ }^{-}$) in air-saturated aqueous solution by singlet-excited APA generated through sensitized TTA using green light. For details, see the text and Section 7 of the ESI. $\dagger$
TTA-based chemical transformation in homogeneous aqueous solution. Providing about $2 \mathrm{eV}$ of reductive power without selfassembly or using microheterogeneous environments, ${ }^{\mathbf{6}, 63-66}$ this process could probably be developed into a versatile method for reductive reactions in water. The only stronger reductant accessible with green light (coinciding with the maximum of the terrestrial solar spectrum) in homogeneous aqueous solution is the hydrated electron, whose generation still requires pulsed lasers, ${ }^{\mathbf{4 6}, \mathbf{6 7}}$ which are more expensive by one order of magnitude compared to the inexpensive cw light source employed in this study.

With the ultimate aim in mind to perform chemical reactions through sensitized TTA with catalytic amounts of both the sensitizer and the acceptor, we did additional irradiation experiments at anthracene concentrations lower by one order of magnitude than in the preceding paragraphs $(1 \mathrm{mM}$, see Section 7 of the ESI $\dagger$ ). Under these conditions, a pseudo-quantitative TTET (which is required for the sensitized TTA mechanism to be efficient) can only be achieved with sensitizers possessing long excited-state lifetimes, i.e., with our dyads. However, for solubility reasons (compare, Section 2.2), we were forced to employ the less reactive anthracene MAMA in our comparative TCA $^{-}$dechlorination experiments (see Section 7 of the ESI for details $\dagger$ ). Although we could not achieve a fully catalytic dechlorination, we observed a three-fold increase of the dechlorination yield when using the dyad RuPy2 instead of Rubpy. These investigations clearly demonstrate that sensitizers with long-lived excited states are important for further improvements of upconversion schemes in water, especially with regard to photochemical reactions at low acceptor concentrations.

Based on our promising results, our study might well stimulate a broader application and a further optimization of aqueous upconversion via sensitized TTA. As our observed insolubilities in the systems RuPy1/APA and RuPy2/APA suggest, the acceptors/annihilators for further studies would need to be cationic when a cationic sensitizer is employed, or their water solubility should be ensured by adding several nonionic polar groups to the hydrophobic parent compounds.

\section{Conclusions}

As has emerged from the two main aspects presented in this work, sensitized triplet-triplet annihilation in pure water - the cheapest and most environmentally friendly solvent - clearly has the potential of being more than a lab curiosity.

First, we were able to improve the overall green-to-violet upconversion efficiency by one order of magnitude compared to the only previously published example of TTA in homogenous aqueous solution. ${ }^{15}$ We have achieved this by combining tailor-made metal complex-arene dyads with highly fluorescent commercial anthracene derivatives. Owing to a triplet reservoir effect, these dyads can be used at very low concentrations, and they pave the way for photon upconversion in homogeneous aqueous solution with attractive quantum yields., ${ }^{7,68}$

Second, we provided the first proof-of-concept for chemical transformations on a laboratory scale via sensitized TTA in 
aqueous media. Our new approach exploits air-saturated water as the reaction medium combined with the pooling of two visible photons to initiate energy-demanding reactions and represents a promising green strategy for photochemical syntheses.

\section{Conflicts of interest}

There are no conflicts to declare.

\section{Acknowledgements}

Financial support provided by the German National Academy of Sciences Leopoldina to CK (postdoctoral fellowship LPDS 201711) and by the Swiss National Science Foundation (grant number 200021_178760) to OSW is gratefully acknowledged.

\section{References}

1 T. N. Singh-Rachford and F. N. Castellano, Coord. Chem. Rev., 2010, 254, 2560-2573.

2 J. Zhao, S. Ji and H. Guo, RSC Adv., 2011, 1, 937-950.

3 V. Gray, D. Dzebo, M. Abrahamsson, B. Albinsson and K. Moth-Poulsen, Phys. Chem. Chem. Phys., 2014, 16, 10345-10352.

4 X. Guo, Y. Liu, Q. Chen, D. Zhao and Y. Ma, Adv. Opt. Mater., 2018, 6, 1700981.

5 J. Zhou, Q. Liu, W. Feng, Y. Sun and F. Li, Chem. Rev., 2015, 115, 395-465.

6 N. Yanai and N. Kimizuka, Chem. Commun., 2016, 52, 53545370.

7 V. Gray, K. Moth-Poulsen, B. Albinsson and M. Abrahamsson, Coord. Chem. Rev., 2018, 362, 54-71.

8 R. R. Islangulov and F. N. Castellano, Angew. Chem., Int. Ed., 2006, 118, 5957-5959.

9 M. Majek, U. Faltermeier, B. Dick, R. Pérez-Ruiz and A. Jacobi von Wangelin, Chem.-Eur. J., 2015, 21, 15496-15501.

10 M. Häring, R. Pérez-Ruiz, A. Jacobi von Wangelin and D. D. Díaz, Chem. Commun., 2015, 51, 16848-16851.

11 M. Rao, K. Kanagaraj, C. Fan, J. Ji, C. Xiao, X. Wei, W. Wu and C. Yang, Org. Lett., 2018, 20, 1680-1683.

12 T. F. Schulze and T. W. Schmidt, Energy Environ. Sci., 2015, 8, 103-125.

13 C. Ye, L. Zhou, X. Wang and Z. Liang, Phys. Chem. Chem. Phys., 2016, 18, 10818-10835.

14 C. A. Parker and C. G. Hatchard, Proc. Chem. Soc., 1962, 386387.

15 K. A. El Roz and F. N. Castellano, Chem. Commun., 2017, 53, 11705-11708.

16 K. El Roz, PhD thesis, North Carolina State University, 2017.

17 P. Anastas and N. Eghbali, Chem. Soc. Rev., 2010, 39, 301312.

18 C. Capello, U. Fischer and K. Hungerbühler, Green Chem., 2007, 9, 927-934.

19 A. J. Howarth, M. B. Majewski and M. O. Wolf, Coord. Chem. Rev., 2015, 282-283, 139-149.
20 M. Montalti, A. Credi, L. Prodi and M. T. Gandolfi, Handbook of photochemistry, CRC/Taylor \& Francis, Boca Raton, 3rd edn, 2006.

21 F. N. Castellano, Acc. Chem. Res., 2015, 48, 828-839.

22 D. S. Tyson and F. N. Castellano, J. Phys. Chem. A, 1999, 103, 10955-10960.

23 G. J. Wilson, A. Launikonis, W. H. F. Sasse and A. W.-H. Mau, J. Phys. Chem. A, 1997, 101, 4860-4866.

24 S. Campagna, F. Puntoriero, F. Nastasi, G. Bergamini and V. Balzani, Top. Curr. Chem., 2007, 280, 117-214.

25 M. M. Cooke, E. H. Doeven, C. F. Hogan, J. L. Adcock, G. P. McDermott, X. A. Conlan, N. W. Barnett, F. M. Pfeffer and P. S. Francis, Anal. Chim. Acta, 2009, 635, 94-101.

26 A. A. Vlcek, E. S. Dodsworth, W. J. Pietro and A. B. P. Lever, Inorg. Chem., 1995, 34, 1906-1913.

27 C. Kerzig and M. Goez, Chem. Sci., 2016, 7, 3862-3868.

28 A. Harriman, M. Hissler, A. Khatyr and R. Ziessel, Chem. Commun., 1999, 735-736.

29 G. J. Wilson, W. H. F. Sasse and A. W.-H. Mau, Chem. Phys. Lett., 1996, 250, 583-588.

30 A. I. Baba, J. R. Shaw, J. A. Simon, R. P. Thummel and R. H. Schmehl, Coord. Chem. Rev., 1998, 171, 43-59.

31 K. Suzuki, A. Kobayashi, S. Kaneko, K. Takehira, T. Yoshihara, H. Ishida, Y. Shiina, S. Oishi and S. Tobita, Phys. Chem. Chem. Phys., 2009, 11, 9850-9860.

32 J. W. Dobrucki, J. Photochem. Photobiol., B, 2001, 65, 136-144.

33 K. J. Morris, M. S. Roach, W. Xu, J. N. Demas and B. A. DeGraff, Anal. Chem., 2007, 79, 9310-9314.

34 A. Son, A. Kawasaki, D. Hara, T. Ito and K. Tanabe, Chem.Eur. J., 2015, 21, 2527-2536.

35 F. N. Castellano and J. R. Lakowicz, Photochem. Photobiol., 1998, 67, 179-183.

36 H. Komatsu, K. Yoshihara, H. Yamada, Y. Kimura, A. Son, S. Nishimoto and K. Tanabe, Chem.-Eur. J., 2013, 19, 19711977.

37 D. Hara, H. Komatsu, A. Son, S. Nishimoto and K. Tanabe, Bioconjugate Chem., 2015, 26, 645-649.

38 E. Roussakis, Z. Li, A. J. Nichols and C. L. Evans, Angew. Chem., Int. Ed., 2015, 54, 8340-8362.

39 O. Johansen, A. W.-H. Mau and W. H. F. Sasse, Chem. Phys. Lett., 1983, 94, 107-112.

40 K. K. Rohatgi-Mukherjee, K. Bhattacharyya and P. K. Das, J. Chem. Soc., Faraday Trans. 2, 1985, 81, 1331.

41 Y. Uchiyama, R. Watanabe, T. Kurotaki, S. Kuniya, S. Kimura, Y. Sawamura, T. Ohtsuki, Y. Kikuchi, H. Matsuzawa, K. Uchiyama, M. Itakura, F. Kawakami and H. Maruyama, ACS Omega, 2017, 2, 3371-3379.

42 A. Yoshimura, M. Z. Hoffman and H. Sun, J. Photochem. Photobiol., A, 1993, 70, 29-33.

43 P. V. Kamat, J. Phys. Chem., 1989, 93, 859-864.

44 A. M. Funston, S. V. Lymar, B. Saunders-Price, G. Czapski and J. R. Miller, J. Phys. Chem. B, 2007, 111, 6895-6902.

45 P. Müller and K. Brettel, Photochem. Photobiol. Sci., 2012, 11, 632.

46 M. Goez, C. Kerzig and R. Naumann, Angew. Chem., Int. Ed., 2014, 53, 9914-9916. 
47 M. Brautzsch, C. Kerzig and M. Goez, Green Chem., 2016, 18, 4761-4771.

48 F. Deng, M. S. Lazorski and F. N. Castellano, Philos. Trans. $R$. Soc., A, 2015, 373, 20140322.

49 F. N. Castellano and C. E. McCusker, Dalton Trans., 2015, 44, 17906-17910.

50 F. Zhong and J. Zhao, Eur. J. Inorg. Chem., 2017, 2017, 51965204.

51 Y. Lu, R. Conway-Kenny, B. Twamley, N. McGoldrick, J. Zhao and S. M. Draper, ChemPhotoChem, 2017, 1, 544-552.

52 Y. Lu, J. Wang, N. McGoldrick, X. Cui, J. Zhao, C. Caverly, B. Twamley, G. M. Ó Máille, B. Irwin, R. Conway-Kenny and S. M. Draper, Angew. Chem., Int. Ed., 2016, 55, 14688-14692.

53 T. N. Singh-Rachford, R. R. Islangulov and F. N. Castellano, J. Phys. Chem. A, 2008, 112, 3906-3910.

54 C. Bohne, E. B. Abuin and J. C. Scaiano, J. Am. Chem. Soc., 1990, 112, 4226-4231.

55 S. Mukherjee and P. Thilagar, Dyes Pigm., 2014, 110, 2-27.

56 B. Xu, Y. Mu, Z. Mao, Z. Xie, H. Wu, Y. Zhang, C. Jin, Z. Chi, S. Liu, J. Xu, Y.-C. Wu, P.-Y. Lu, A. Lien and M. R. Bryce, Chem. Sci., 2016, 7, 2201-2206.

57 Higher sensitizer concentrations are required to reach the linear regime of the excitation intensity dependence, ${ }^{58}$ which would be important for application-related studies, but not for mechanistic investigations.

58 A. Haefele, J. Blumhoff, R. S. Khnayzer and F. N. Castellano, J. Phys. Chem. Lett., 2012, 3, 299-303.

59 C. Kerzig and M. Goez, Phys. Chem. Chem. Phys., 2014, 16, 25342-25349.

60 P. Wardman, J. Phys. Chem. Ref. Data, 1989, 18, 1637-1755.

61 X. Liu, J. Zhong, L. Fang, L. Wang, M. Ye, Y. Shao, J. Li and

T. Zhang, Chem. Eng. J., 2016, 303, 56-63.

62 R. Schmidt, K. Schaffner, W. Trost and H. D. Brauer, J. Phys. Chem., 1984, 88, 956-958.

63 H. Kouno, T. Ogawa, S. Amemori, P. Mahato, N. Yanai and N. Kimizuka, Chem. Sci., 2016, 7, 5224-5229.

64 S. H. C. Askes, P. Brodie, G. Bruylants and S. Bonnet, J. Phys. Chem. B, 2017, 121, 780-786.

65 A. Turshatov, D. Busko, S. Baluschev, T. Miteva and K. Landfester, New J. Phys., 2011, 13, 083035.

66 J. Park, M. Xu, F. Li and H.-C. Zhou, J. Am. Chem. Soc., 2018, 140, 5493-5499.

67 R. Naumann, C. Kerzig and M. Goez, Chem. Sci., 2017, 8, 7510-7520.

68 S. Hoseinkhani, R. Tubino, F. Meinardi and A. Monguzzi, Phys. Chem. Chem. Phys., 2015, 17, 4020-4024. 